\title{
An event-based account of coordination stability
}

\author{
REBECCA M. C. SPENCER \\ University of California, Berkeley, California \\ ANDRAS SEMJEN \\ Centre National de la Recherche Scientifique, Marseille, France \\ and \\ STEPHANIE YANG and RICHARD B. IVRY \\ University of California, Berkeley, California
}

\begin{abstract}
Constraints underlying bimanual coordination have traditionally been explained by dynamic interactions between the effectors. However, the present experiments demonstrate that a fundamental constraint on bimanual performance is the manner in which task goals are represented. In Experiment 1, participants vocalized during in-phase and anti-phase bimanual movements. As expected, most participants spontaneously exhibited temporal coupling between the manual and vocal responses. However, the form of coupling differed for the in-phase and anti-phase conditions. For anti-phase movements, there was a strong bias to produce two vocalizations per cycle; for in-phase movements, participants were equally likely to produce one or two vocalizations per cycle. We hypothesized that the spontaneous vocalizations probed the cognitive representation of the task, and the results indicated that anti-phase movements did entail a more complex event structure than in-phase movements did. In Experiment 2, we manipulated the event structure by having participants vocalize either once or twice per hand cycle. As predicted, coordination stability was reduced when the event structure was more complex.
\end{abstract}

The coordination of rhythmic bimanual movements has served as a paradigmatic task for exploring constraints underlying motor control. The focal point of this work has been the relative stability of in-phase movements in comparison with anti-phase movements (Carson, 1993; Kelso, 1995). For in-phase movements, the two limbs follow mirror-symmetric trajectories; for anti-phase movements, the trajectories are asymmetric. When performed repetitively at increasing movement frequencies, the anti-phase pattern is difficult to maintain and spontaneous transitions to the in-phase pattern frequently occur.

The greater stability of in-phase movements in comparison with anti-phase movements has been attributed to constraints associated with motor planning and execution. One source of constraint is a preference to activate homologous muscles (Cohen, 1971). If the same effectors are used, in-phase movements involve homologous muscles whereas anti-phase movements involve opposing muscles. One neural instantiation of this constraint emphasizes the role of nondecussating fibers in the cortico-

We dedicate this paper to the memory of Andras Semjen. We are grateful to Sophie Ghiam for her assistance in collecting the data in Experiment 1 and to Steven Keele and Matthew Brett for comments on the manuscript. This work was supported by NIH Grants NS048012, NS30256, NS17778, and NS40813. Correspondence should be addressed to R. M. C. Spencer, Department of Psychology, University of California, 3210 Tolman Hall No. 1650, Berkeley, CA 94270-1650 (e-mail: rspencer@socrates.berkeley.edu). spinal tract (Cattaert, Semjen, \& Summers, 1999). The signals from the dominant, crossed fibers would be facilitated by signals from ipsilateral fibers during in-phase movements. During anti-phase movements, these signals would conflict.

The preference for in-phase movements, however, extends to conditions in which two actions are produced by nonhomologous muscles (Kelso \& Jeka, 1992; Mechsner, Kerzel, Knoblich, \& Prinz, 2001) or when movements are made by two people (see, e.g., Schmidt, Carello, \& Turvey, 1990). Results such as these have been interpreted as providing evidence that the preference for symmetric patterns arises at more abstract levels described by the task dynamics (see Turvey, 1990). Formally, these dynamics have been characterized as resulting from the continuous interactions of coupled oscillators (e.g., Haken, Kelso, \& Bunz, 1985; Yaminishi, Kawato, \& Suzuki, 1980). Inphase patterns are stable over a wide range of frequencies because the coupling between the oscillators reinforces the patterns' individual, stable dynamics. Anti-phase patterns break down at high frequencies (i.e., fast movements) because the coupling constraint biases the two oscillators to become synchronized.

In the present study, we explore a novel account of the constraints on rhythmic, bimanual movements. Rhythmic movements can be represented as a series of salient events that define the temporal goals of a task (Ivry, Spencer, Zelaznik, \& Diedrichsen, 2002; Spencer, Zelaznik, Diedrichsen, \& Ivry, 2003). We refer to this representation as a 
temporal event structure. Like the dynamic systems perspective, this representation is abstract in that the events define temporal ratios between successive events. The instantiation of this representation involves the mapping of these temporal goals onto salient events in the movement. Consider a unimanual task involving flexion and extension of the wrist. If the movements were made on a table surface, the temporal goals might be realized by timing the intervals between successive table taps (see Billon, Semjen, \& Stelmach, 1996). If the movements were made in the air, the salient events might correspond to the perceived onset of each cycle. We hypothesize that, for such tasks, an internal timing system provides the temporal signals to control the transition from one event to the next. ${ }^{1}$

The temporal event structure for in-phase, bimanual movements would be the same as for unimanual movements. For example, if the temporal goals during unimanual tapping were realized by timing the interval between one tap and the next, the same event structure would suffice during bimanual tapping, with the additional task goal that the two taps be synchronized. Similarly, if the movements were made in the air, the onset points for each flexion phase would be synchronized (Carson, 1996).

In contrast, a simple generalization of the unimanual event structure may not be appropriate for anti-phase movements. In the table-tapping and wrist flexion examples the salient points in an anti-phase movement cycle (i.e., the taps and flexion onsets) would be temporally offset for the two hands. If the temporal goals were achieved by referencing these points, then a more complex event structure would be adopted: each cycle would now entail two events, one corresponding to the salient point in the cycle for the left hand and a second for the corresponding point for the right hand. If we assume that pattern stability is inversely related to the complexity of the temporal event structure, we would expect that anti-phase movements would be less stable than in-phase movements. The following experiments demonstrate the viability of this framework for understanding constraints on bimanual coordination.

\section{EXPERIMENT 1}

Experiment 1 was designed to probe the temporal event structure of in-phase and anti-phase bimanual rhythmic movements. To this end, participants were instructed to perform either the in-phase or the anti-phase pattern while repetitively saying the word "ba." We took care to provide no instructions regarding the linkage between the vocalizations and the manual movements. Nonetheless, we expected that the participants would exhibit temporal coupling between the two tasks (Kelso, 1995). Our interest was in the rate at which they spontaneously chose to vocalize. Given the superficial similarity of the two movement patterns, one might expect participants to coordinate their vocalizations and movements in a similar manner for the in- and anti-phase conditions. However, given the assumption that the vocalizations would be constrained by the underlying temporal event structure, we predicted that participants would choose to say "ba" twice per cycle in the anti-phase condition and once per cycle in the in-phase condition. Support for this prediction would be consistent with the hypothesis that anti-phase movements entail a more complex temporal event structure than in-phase movements. The results from a pilot version of this experiment were summarized in Ivry, Diedrichsen, Spencer, Hazeltine, and Semjen (2004).

\section{Method}

Participants. Twenty-four right-handed students (18-26 years old) participated in Experiment 1. The procedures were approved by the Institutional Review Board of the University of California, Berkeley. Informed consent was obtained prior to testing.

Task and design. The participants rested their elbows on a table and produced flexion and extension movements of the wrists. The forearms were elevated above the table to eliminate tactile cues to the hands. The participants were randomly assigned to either the inphase $(n=12)$ or anti-phase $(n=12)$ group. The in-phase group was instructed to move the hands "up together and down together"; the anti-phase group was instructed to "move one hand up when moving the other hand down" (Figure 1). The experimenter demonstrated the appropriate posture and produced a single flexion-extension movement. Beyond this, care was taken to minimize instructions.

The experiment was divided into two parts, each consisting of six blocks of 10 trials. Target cycle durations for five of the blocks were $1,200,1,000,750,500$, or $300 \mathrm{msec}$, and one block had instructions to "move at a comfortable pace." Half of the participants in each group performed the blocks in ascending order of duration with the spontaneous rate last. For the other participants, the blocks were arranged in descending order of duration with the spontaneous rate performed first.

In each block that included target duration cycles, the first five trials consisted of seven movement cycles. The mean cycle duration was displayed on the computer monitor after each of these trials. If the mean rate was within $25 \mathrm{msec}$ of the goal rate, the participant was told to maintain the adopted rate. If the mean rate was $25-100 \mathrm{msec}$ faster or slower than the target rate, the experimenter instructed the participant to move "a little faster or a little slower." If the mean rate differed from the target rate by more than $100 \mathrm{msec}$, the instructions were modified to "move much faster or much slower." No feedback was provided on the blocks with spontaneous rates.

For the last five trials of each block, the participant produced 20 movement cycles at the instructed rate. During these trials, the participant was instructed to "say 'ba' repeatedly as you move." In the second half of the experiment, the instructions were to "say 'ba' in synchrony with your hand movements." 2 No instructions were provided regarding synchronizing the vocalizations and the hand movements, nor did the experimenter provide any demonstrations.

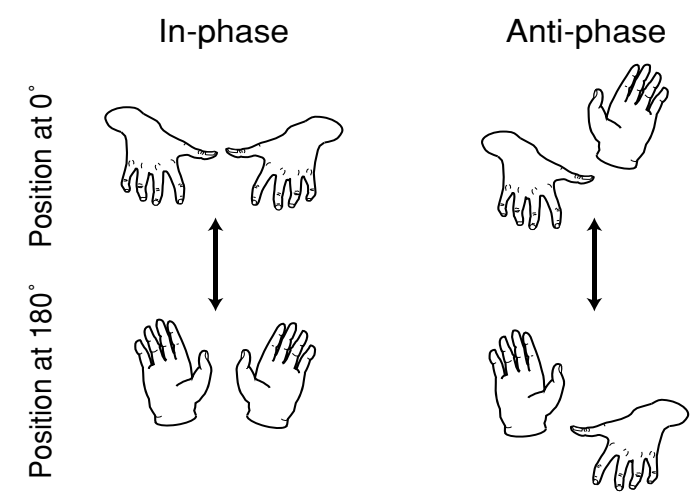

Figure 1. Hand movements produced in Experiment 1. 
Feedback was eliminated during these trials. We also did not utilize a metronome at any point in the experiment, since this would likely have influenced the conceptualization of the event structure. In particular, we did not want to bias the participants to vocalize at the rate of the metronome.

Between blocks, participants said one of three tongue twisters as fast as possible for $30 \mathrm{sec}$. This task provided a ruse for the inclusion of the vocalizations, with participants likely to think the tasks were related. Additionally, by having the tongue twisters spoken rapidly, we expected to reduce carryover effects of the vocal-manual coupling from one block to the next.

Data acquisition and analysis. Kinematic data were collected with an Ascension miniBIRD tracking system (138-Hz sampling rate). One $8 \times 8 \times 12$-mm marker was attached to each index finger.
Cycle durations were computed as the time between local maxima in the $z$-dimension (parallel to the body axis). Vocalizations were digitally recorded with a microphone at a sampling rate of $22.05 \mathrm{kHz}$. Voice cycle durations were measured from the time of voice onset in one cycle to the time of voice onset in the next cycle.

\section{Results and Discussion}

Participants were accurate in matching the target cycle durations on the last five trials in which vocalizations were added and feedback on cycle duration was no longer provided. The mean cycle durations for the $1,200,1,000$, 750,500 , and $300 \mathrm{msec}$ conditions were 1,169, 1,034, 801, 602 , and $384 \mathrm{msec}$, respectively, for the in-phase group

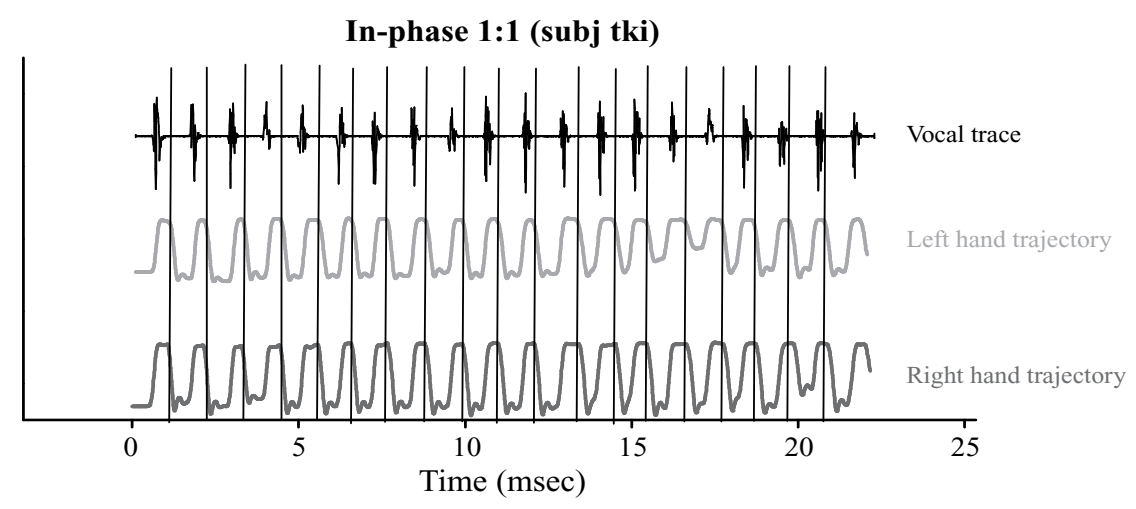

In-phase 2:1 (subj tbi)

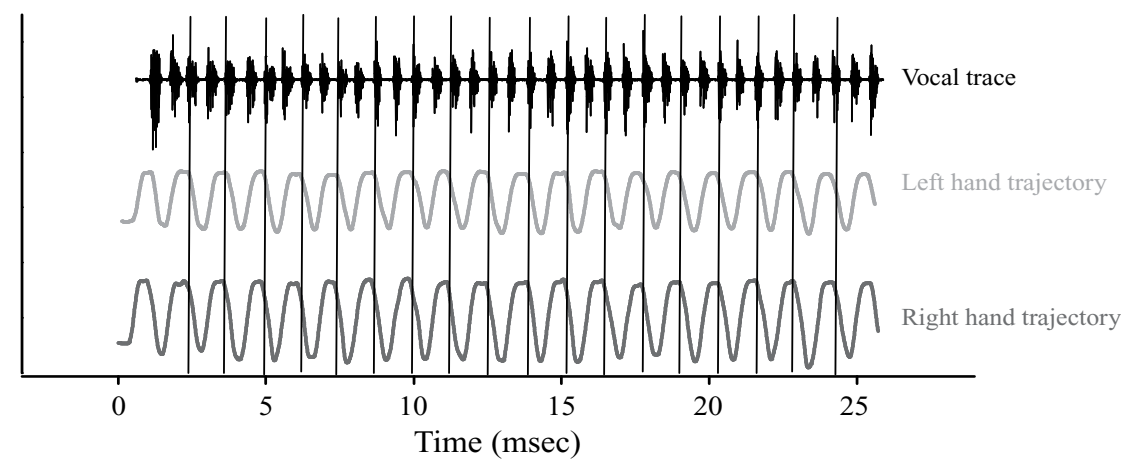

Anti-phase 2:1 (subj mla)

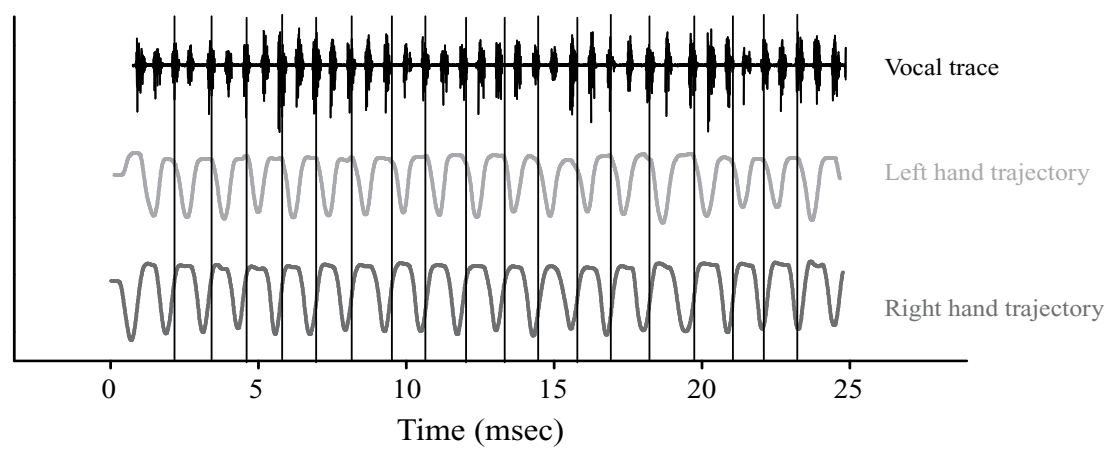

Figure 2. Representative trials of the three predominant strategies adopted in Experiment 1. For each trial, the vocal trace indicates the raw waveform obtained from the microphone and the manual trajectories (displacement in the $z$-dimension). Vertical lines indicate the time at which the analysis algorithm identified onset of a movement cycle for the left hand. 
and 1,191, 1,011, 781, 539, 358 msec, respectively, for the anti-phase group. In the spontaneous rate condition, participants produced a range of movement rates. Those who performed the spontaneous rate block first tended to adopt faster cycle durations $(M=752 \mathrm{msec})$ than those who performed this block last $(M=1,029 \mathrm{msec})$. Instructions had minimal effect since most participants synchronized their vocalizations with their hand movements regardless of task instructions. Three participants (one in-phase; two anti-phase) did not synchronize vocalizations with hand movements in the first half of the experiment. These participants adopted a single vocalization rate regardless of the hand movement rate. For example, one participant vocalized at a rate of just over $2 \mathrm{~Hz}$ in all blocks and thus the hand movements and vocalizations were uncoupled. Data from these three participants were excluded from the subsequent analyses.

Figure 2 displays representative trials for the three most commonly observed patterns. As the traces of the hand kinematics show, participants maintained a consistent phase relationship between the hands. Similarly, the timing of the vocalizations with respect to the hand movements remained stable. Our main interest was in the qualitative relationship between the rate of the instructed hand movements and the rate of the spontaneous vocalizations. To this end, we calculated the ratio of voice cycle duration to hand cycle duration. This calculation was performed trialby-trial and an average was calculated for each block. Averaging was justified given that the ratios remained consistent within a block. Ratios were classified as $1: 1$ if they fell between $0.9: 1$ and $1.1: 1$ and as $2: 1$ when they were between 1.9:1 and 2.1:1. The ratio for all of the blocks fell within one of these ranges.

Across all of the duration rates, the 10 participants in the anti-phase group spontaneously vocalized twice per hand cycle (Table 1). The only exception was that at the fastest rate, one of the participants switched to one vocalization per cycle. In contrast, only 3 of the participants in the in-phase group consistently vocalized twice per hand cycle. Five spontaneously opted to vocalize once per cycle at all movement rates. The remaining 3 participants produced two vocalizations per cycle at the slower rates and switched to one vocalization per cycle at the faster rates. A chi-square test of the vocal-manual ratio (always $1: 1$, always $2: 1$, or mixed) for the two groups verified that the in- and anti-phase groups differed in how they chose to synchronize the vocalization and manual tasks $\left[\chi^{2}(2, N=\right.$ 21) $<8.97, p=.03]$.

As expected, the majority of the participants spontaneously coupled vocalizations with hand movements (Kelso, 1995). Assuming that the vocalizations provided a probe on the underlying temporal event structure, the present results indicate that anti-phase movements generally entailed a more complex event structure than in-phase movements. Participants moving anti-phase almost always produced two vocalizations per cycle, in accordance with the hypothesis that these movement patterns are demarcated by two salient events per cycle. The results were more
Table 1

Numbers of Participants Adopting Either a 1:1 or a 2:1 Vocal:Manual Ratio for the In-Phase and Anti-phase Groups in Experiment 1

\begin{tabular}{cccr}
\hline & & \multicolumn{2}{c}{ Vocal:Manual } \\
& & Ratio & $2: 1$ \\
\cline { 3 - 4 } Group & Rate $(\mathrm{msec})$ & $1: 1$ & $\mathbf{6}$ \\
In-phase & SPO & 5 & $\mathbf{6}$ \\
& 1,200 & 5 & $\mathbf{6}$ \\
& 1,000 & 5 & $\mathbf{6}$ \\
& 750 & 5 & 4 \\
Anti-phase & 500 & $\mathbf{7}$ & 3 \\
& 300 & 0 & $\mathbf{1 0}$ \\
& SPO & 0 & $\mathbf{1 0}$ \\
& 1,200 & 0 & $\mathbf{1 0}$ \\
& 1,000 & 0 & $\mathbf{1 0}$ \\
& 750 & 0 & $\mathbf{1 0}$ \\
& 500 & 1 & $\mathbf{9}$
\end{tabular}

Note-The predominant ratio is in boldface. Three participants failed to adopt either the $1: 1$ or the $2: 1$ rate and are excluded from the table.

ambiguous for participants in the in-phase group. Half of the participants vocalized only once per cycle, suggesting that they conceptualized a single salient event per cycle. The other half of the participants adopted a 2:1 ratio at the slower speeds. Interestingly, at faster rates, some of these participants shifted to a 1:1 ratio. This shift suggests that participants in the in-phase group preferred a simpler event representation as movement frequency increased.

\section{EXPERIMENT 2}

In Experiment 1, the vocalization task was used to probe the temporal representation of rhythmic movements. The results are consistent with the hypothesis that in- and antiphase movements may entail different temporal event structures. In Experiment 2, we sought to test the corollary hypothesis that the stability of these rhythmic movements may be related to the complexity of the event structure.

Participants again vocalized while making repetitive wrist movements. However, they were explicitly instructed to produce either one or two vocalizations with each hand cycle while increasing hand movement frequency. Antiphase movements produced at increasing cycle frequencies become unstable (see, e.g., Kelso, 1995). We assumed that the instructed vocalization pattern would influence the event structure associated with the hand movements, which would in turn influence the stability of the hand movements. In particular, we hypothesized that an imposed 2:1 ratio would lead to a more complex temporal event structure than an imposed 1:1 ratio. We predicted that a behavioral consequence of this complexity manipulation would be manifest in the stability of the hand movement pattern - that is, performance would be more stable when the movements reflected a simpler event structure.

This hypothesis leads to a counterintuitive prediction for anti-phase movements. In Experiment 1, participants spontaneously adopted a 2:1 ratio when moving the hands anti-phase. Given this, one might expect that performance 
would be more stable in the two-vocalizations-per-cycle condition than in the one-vocalization-per-cycle condition, since the latter imposes an event structure that is discordant with the spontaneous representation. However, the temporal event structure hypothesis leads to the opposite prediction. We assumed that by requiring only one vocalization per cycle, we would induce a simpler event structure than that which was spontaneously adopted. Thus, we predicted that performance would be more stable in the one-vocalization-per-cycle condition than in the twovocalizations-per-cycle condition.

We also included a no-vocalization condition. This condition served two purposes. First, it allowed us to assess whether the imposition of a secondary task affected performance in nonspecific ways. The vocalization task may have taxed attentional resources. In this case, we would expect performance to be most stable in the no-vocalization condition. Second, the no-vocalization condition provided a further test of our assumptions concerning the relationship between the vocalization task and the temporal event structure. Given the results of Experiment 1, we assumed that the event structure here would match that induced by the 2:1 ratio. Thus, we would expect performance to be similar in the no-vocalization and the two-vocalizations conditions. Moreover, the complexity hypothesis predicted that the one-vocalization condition would also be more stable than the no-vocalization condition given that the former would induce a simpler event representation.

As noted previously, a hallmark of the instability of anti-phase movements is their tendency to undergo, at fast movement rates, a transition to in-phase movements. We focused on this measure of stability in Experiment 2, comparing the times at which phase transitions occurred for the three conditions.

\section{Method}

Participants. Sixteen right-handed university students (18-26 years old) were tested.

Tasks and design. All movements involved abduction and adduction of the wrists with the palm downward. The participants were divided into two groups of 8 . The in-phase group was instructed to move the hands "in together and out together" and the anti-phase group was instructed to move the hands "to the left together and to the right together" (Figure 3). ${ }^{3}$ Both groups performed three conditions: hand movements alone, hand movements when instructed to say "ba" once per hand cycle, and hand movements when instructed to say "ba" twice per hand cycle. There were two blocks of six trials for each condition with the order randomized.

The participants began each trial at a comfortable hand movement rate. To obtain a range of movement rates, the experimenter displayed a sign that said "FASTER" after every 10th hand cycle. The participants were informed that there would be four movement rate segments per trial and that they should increase their rate in each segment so that they would be going at a fast rate by the end of each trial.

Data acquisition and analysis. Cycle durations were computed as the time between local maxima in the $x$-dimension (horizontal plane) for the left hand (far right point) and local minima in the $x$ dimension for the right hand data (far left point).

\section{Results and Discussion}

Relative phase, the position of one hand with respect to the other in the movement cycle, was measured for the

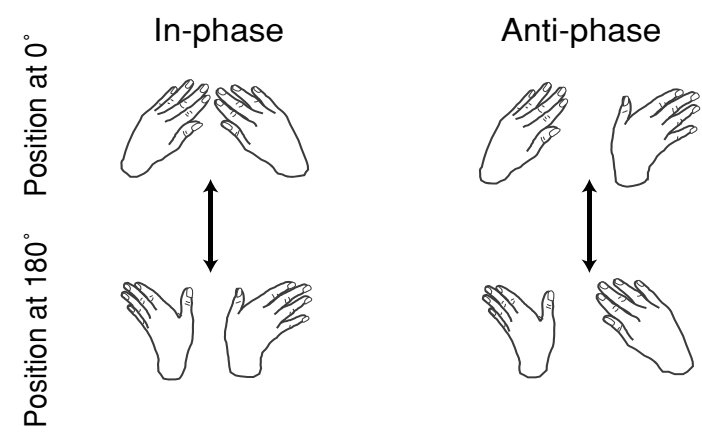

Figure 3. Hand movements produced in Experiment 2.

limbs. If the hands were moving perfectly in the in-phase mode, the relative phase would be $0^{\circ}$; perfect anti-phase coordination would have a relative phase of $180^{\circ}$. Participants were able to maintain the target relative phase in the in-phase condition; no transitions from in-phase to antiphase movements were observed (relative phase values were consistently below $45^{\circ}$ ). In contrast, a loss of pattern stability was observed on many anti-phase trials as movement rate increased, consistent with previous studies (e.g., Kelso, Scholz, \& Schoner, 1986).

While our instructions were designed to induce step changes in movement rates, the actual changes tended to be continuous. Given this, the movement rate for each cycle was calculated and sorted into $50 \mathrm{msec}$ bins. The relative phase between hands was calculated for trial segments corresponding to these bins. When relative phase was $\leq 45^{\circ}$, the movements were classified as "in-phase"; when relative phase was $\geq 135^{\circ}$, the movements were classified as "anti-phase." Cycles that did not fall within these criteria were classified as "other."

For the anti-phase condition, a rapid decrease in the percentage of anti-phase cycles was observed for cycle durations shorter than $550 \mathrm{msec}$, main effect of rate $\left[F(10,216)=16.4, p<.001, \eta^{2}=.45\right]$ (Figure 4A). Notably, this decrease varied as a function of the vocalization requirement $\left[F(2,216)=3.61, p=.03, \eta^{2}=.02\right]$. When vocalizing twice per cycle, participants were more likely to successfully maintain the anti-phase pattern than when they produced either one or no vocalizations per cycle. While Figure 4A suggests that this effect was most pronounced at the faster rates, the condition $\times$ rate interaction was not reliable $[F(20,216)<1]$.

An alternative way to look at stability is to focus on the point of pattern transition. We identified the movement rate at which participants failed to maintain the antiphase pattern (relative phase $<135^{\circ}$ for more than two consecutive cycles). This value was identified for each trial in which such transitions occurred, ${ }^{4}$ and then averaged across trials for each condition for each participant. The number of vocalizations per hand cycle influenced the hand cycle duration associated with this transition (Figure 4B). Participants were able to maintain the antiphase pattern at faster rates in the one-vocalization condition than in the two-vocalization condition $[F(1,14)=$ 
5.66, $\left.p=.03, \eta^{2}=.30\right]$. A similar comparison of the two- and no-vocalization conditions was marginally significant $\left[F(1,14)=4.33, p=.057, \eta^{2}=.25\right]$. Thus, while transitions to in-phase movements were observed in all anti-phase conditions, participants better maintained the anti-phase pattern when vocalizing once per cycle than in the other conditions.

Finally, we examined whether vocalization frequency influenced stability during the trial epochs in which participants maintained the anti-phase pattern. As in the other analyses, relative phase variability was influenced by the vocalization requirements, as shown in Figure 4C [twovs. one-vocalization conditions: $F(1,110)=5.2, p<.01$, $\eta^{2}=.27$; two- vs. no-vocalization condition: $F(1,110)=$ $\left.12.9, p<.01, \eta^{2}=.07\right]$. Variability increased with rate for all three conditions. Notably, this factor interacted with group in the comparison of the two- and no-vocalization conditions $\left[F(7,110)=2.15, p=.05, \eta^{2}=.08\right]$; the interaction only approached significance in the comparison of the two- and one-vocalization conditions $[F(7,110)=$ $0.1]$. Thus, even during the epochs in which the anti-phase pattern was maintained, participants were more stable when vocalizing once per cycle.

In sum, coordination stability was influenced by vocalization requirements. Given the results of Experiment 1, we assumed that the no-vocalization anti-phase task would entail a temporal event structure similar to that for the two-vocalization anti-phase task. The results for these two conditions are in accord with this assumption. Moreover, the results are consistent with the hypothesis that pattern stability is constrained by the manner in which the event structure is represented. Requiring one vocalization per hand cycle led to improved pattern stability in comparison with that found in the two-vocalization and no-vocalization conditions. We assumed that the one-vocalization condition would impose a simpler temporal event structure, even though it diverged from the spontaneous event structure associated with anti-phase movements.

\section{GENERAL DISCUSSION}

The relative stability of in-phase as opposed to antiphase movements has traditionally been described in terms of the coordination dynamics of coupled oscillators (see Schoner \& Kelso, 1988). Such dynamics have been assumed to reflect various sources, such as properties of the motor system (e.g., preference for homologous muscles) or the influence of the task environment. For example, coordination is stabilized when the movements are paced by a metronome (Fink, Foo, Jirsa, \& Kelso, 2000) or when haptic cues are provided (Kelso, Fink, DeLaplain, \& Carson, 2001). Such effects can be viewed as reflecting the interaction of task constraints on coordination dynamics.

While the dynamic systems perspective has provided elegant descriptions of pattern stability, the present experiments provide a process-based account of constraints underlying bimanual coordination. A central feature of this account is that the constraints underlying the coordination of repetitive movements may not reflect continuous interactions, as would be suggested by mechanisms such as coupled oscillators. Rather, we propose that the primary constraints arise from the manner in which the task

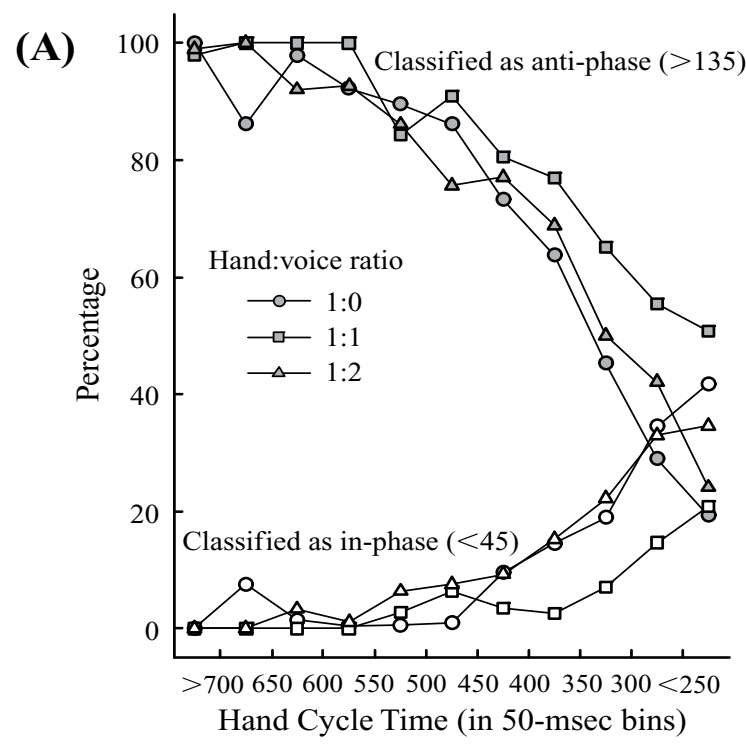

(B)

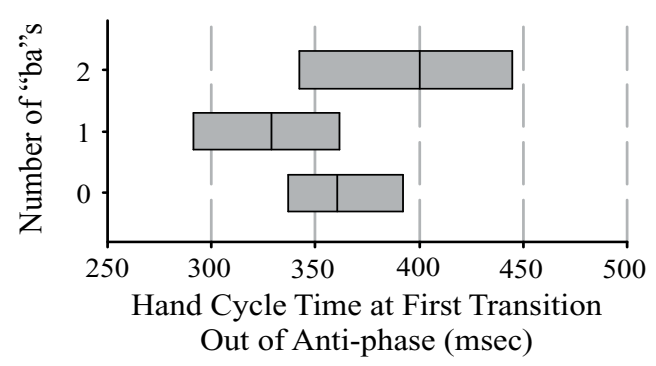

(C)

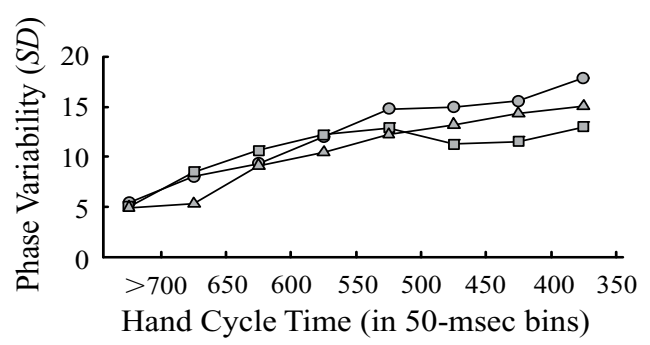

\footnotetext{
Figure 4. Results from Experiment 2. (A) Percentage of movements classified as in-phase or anti-phase as a function of movement rate in the anti-phase conditions (see text). Perfect performance would result in $100 \%$ of the cycles' being classified as anti-phase. Performance deteriorates for all three groups. (B) Hand cycle frequency corresponding to the first phase transition in the anti-phase conditions. Each box represents a $75 \%$ confidence interval; the error bars represent the $95 \%$ confidence interval, and the vertical line splitting each box represents the median. (C) The standard deviation of relative phase for cycles prior to the phase transitions. The two bins with the fastest rates, shown in panel $A$, are not included in panel $C$ because there were so few trials in which anti-phase performance was stable for an extended period of time at these rates.
} 
goals are represented. For repetitive movements, the goals entail an abstract rhythmic representation, instantiated as a series of temporally defined events.

Experiment 1 used a novel probe to assess the internal representation of the in-phase and anti-phase patterns. Under minimal instruction, participants performing anti-phase movements were more likely to vocalize twice per cycle than participants in the in-phase condition. We assume that this difference reflects the fact that the spontaneous representation of the rhythm associated with anti-phase movements entailed a more complex event structure, with two salient events per hand cycle.

In Experiment 2, we tested the relationship between event structure complexity and pattern stability. By varying the number of vocalizations per cycle, we assumed that we would manipulate the complexity of the rhythmic representation. When a more complex representation composed of two events per cycle was required, anti-phase movements became less stable, with phase transitions occurring earlier, and with greater relative phase variability than in the condition in which the same movement pattern was produced with a vocalization requirement that promoted a simpler, one-event-per-cycle representation. Interestingly, although the two-event representation was spontaneously associated with anti-phase movements, it was not conducive for optimal performance.

These experiments rest on the assumption that vocalizations can be used to probe (as in Experiment 1) or manipulate (as in Experiment 2) the temporal event structure. It is important to consider other ways in which the vocalization task might have influenced performance. When people are asked to synchronize vocal and manual responses, the vocal responses tend to lead the manual responses (Chang \& Hammond, 1987). ${ }^{5}$ This raises the question of whether the vocalization task in Experiment 1 can be considered simply a "probe." We expected that the tasks would interact in significant ways; nonetheless, we observed a qualitative difference in the spontaneous choice of vocalization frequency in the anti- and in-phase conditions. Moreover, the choice of a 2:1 or 1:1 ratio was apparent as soon as the participants began each trial. These observations strongly support the notion that the vocalization pattern was constrained by the manner in which the manual task was represented.

The vocalization requirements may also have had an attentional effect. The vocalization task could have reduced the amount of attention available for the manual task. This hypothesis fails, however, to account for the finding in Experiment 2 that participants' performances were most stable when vocalizations occurred once per cycle in the anti-phase condition. Furthermore, performance was similar in the no- and two-vocalization conditions. The latter result is consistent with the assumption that the two-vocalization condition matched the spontaneous representation of anti-phase movements. By this view, it would be reasonable to assume that the one-vocalization condition was the most attentionally demanding given that it failed to conform to the spontaneous event structure. Of course it may be that the vocalization task did not im- pose additional attentional demands because the vocal and manual tasks were coupled. Other studies have shown that rhythmic performance can be stabilized by the inclusion of salient external (Fink et al., 2000; Kelso et al., 2001) or internal (Ivry \& Richardson, 2002) events.

Indeed, the event representation hypothesis provides a novel account of many factors shown to influence coordination dynamics. Extrinsic constraints such as haptic touch (Kelso et al., 2001) or the presence of a metronome (Carson, 1995; Fink et al., 2000) are salient features that can define the event structure. For example, a metronome does not provide a continuous, oscillating signal, but rather produces salient events that define the task goals. We would expect that the metronome would serve to reinforce and sharpen an event-based representation. As such, performance should become more stable.

The effects of intrinsic constraints can also be understood from an event-based framework. Scholz and Kelso (1990) report that when participants were instructed to intentionally switch from in-phase to anti-phase movements, they were able to maintain an anti-phase pattern at higher movement frequencies. We posit that the initial inphase pattern would involve a representation of one event per cycle. This representation would then be maintained when the participant intentionally switched to the antiphase mode. As shown in Experiment 2, this representation would delay the onset of phase transitions.

An appealing feature of the event representation theory is that it is applicable to a broad range of motor behaviors. Consider a unimanual finger-tapping task in which participants repeatedly vocalize while maintaining a consistent tapping rate. Temporal variability is considerably greater when vocalizations are asynchronous with the tapping cycles than when they are synchronous with them (Klapp, 1981; see also Chang \& Hammond, 1987). Asynchronized vocalization would conflict with the temporal event structure associated with the tapping.

While this unimanual task might be accounted for by the interactions of coupled oscillators (with the vocalizations serving as the second oscillator), deviations in the production of polyrhythms are similar when the movements are produced by either one or two hands (Semjen \& Ivry, 2001). It is unclear how a coupled oscillator model could account for this result. Rather, a more parsimonious account is that these deviations reflect a preference for simple rhythmic ratios (e.g., 1:1, 2:1), which holds regardless of the number of effectors. The processing limitations highlighted by our event-based account provide a natural link between perception, action, and cognition, given that similar constraints would be expected to influence our perception of patterned events as well as the manner in which we produce actions guided by these representations.

We do not wish to suggest that control is limited to the cycle points marked as salient events. For example, in unimanual tapping, some process must control the extension of the finger, even if the event representation specifies the time between successive flexion onsets (or taps). Similarly, for bimanual movements, some process is required to en- 
sure that the trajectory for each hand is realized, regardless of whether a one- or two-event representation is adopted. The event-representation account is directed at a high level of control, one that ensures that the goals for the action are realized. The notion of goal-based constraints has proven useful in understanding limitations in the coordination of bimanual movements (see, e.g., Franz, Zelaznik, Swinnen, \& Walter, 2001; Mechsner et al., 2001) and, indeed, is central to many theories of motor control (see, e.g., Bernstein, 1967; Hommel, Müsseler, Aschersleben, \& Prinz, 2001; Turvey, 1990). Within these theoretical accounts, additional levels of control are proposed to account for the production of the actual movements.

In a similar vein, while the present experiments emphasized the role of event representations as critical constraints influencing the stability of rhythmic movements, we do not claim that they are the sole constraints determining pattern stability. Biomechanics surely impose their own set of constraints. For instance, phase transitions occur earlier for wrist abduction and adduction movements than for wrist flexion and extension movements (see note 3 ). At a more cognitive level, movements with compatible spatial codes are easier to perform than those in which such codes are incongruent (Franz, Zelaznik, \& McCabe, 1991; Semjen, Summers, \& Cattaert, 1995), independent of biomechanics. Such codes may be perceptual in nature (Mechsner, 2004a, 2004b; Mechsner et al., 2001; see also Kunde \& Weigelt, 2005), and/or they may reflect interactions associated with response selection (Ivry et al., 2004). Manipulations of attentional resources can also influence pattern stability (see, e.g., Monno, Temprado, Zanone, \& Laurent, 2002). Understanding the representational basis of these various sources of constraint is essential for the development of process-based models.

\section{REFERENCES}

BERNSTEIN, N. A. (1967). The coordination and regulation of movement. London: Pergamon.

Billon, M., Semjen, A., \& Stelmach, G. E. (1996). The timing effects of accent production in periodic finger-tapping sequences. Journal of Motor Behavior, 28, 198-210.

Carson, R. G. (1993). Manual asymmetries: Old problems and new directions. Human Movement Science, 12, 479-506.

CARson, R. G. (1995). The dynamics of isometric bimanual coordination. Experimental Brain Research, 105, 465-476.

CARson, R. G. (1996). Neuromuscular-skeletal constraints upon the dynamics of perception-action coupling. Experimental Brain Research, 110, 99-110.

Cattaert, D., Semuen, A., \& Summers, J. J. (1999). Simulating a neural cross-talk model for between-hand interference during bimanual circle drawing. Biological Cybernetics, 81, 343-358.

Chang, P., \& Hammond, G. R. (1987). Mutual interactions between speech and finger movements. Journal of Motor Behavior, 19, 265 274.

Cohen, L. (1971). Synchronous bimanual movements performed by homologous and non-homologous muscles. Perceptual \& Motor Skills, 32, 639-644.

Fink, P. W., Foo, P., Jirsa, V. K., \& Kelso, J. A. S. (2000). Local and global stabilization of coordination by sensory information. Experimental Brain Research, 134, 9-20.

Franz, E. A., ZelazNiK, H. N., \& McCabe, G. (1991). Spatial topological constraints in a bimanual task. Acta Psychologica, 77, 137-151.
Franz, E. A., Zelaznik, H. N., Swinnen, S., \& Walter, C. (2001). Spatial conceptual influences on the coordination of bimanual actions: When a dual task becomes a single task. Journal of Motor Behavior, 33, 103-112.

Haken, H., Kelso, J. A. S., \& Bunz, H. (1985). A theoretical model of phase transitions in human hand movements. Biological Cybernetics, 51, 347-356.

Hommel, B., Müsseler, J., Aschersleben, G., \& Prinz, W. (2001). The theory of event coding: A framework for perception and action. Behavioral \& Brain Sciences, 24, 869-937.

IVry, R. B., Diedrichsen, J., Spencer, R. M. C., Hazeltine, E., \& SemJEN, A. (2004). A cognitive neuroscience perspective on bimanual coordination and interference. In S. P. Swinnen \& J. Duyens (Eds.), Interlimb coordination (pp. 259-295). Norwell, MA: Kluwer Academic.

IVRY, R. B., \& RICHARDSON, T. C. (2002). Temporal control and coordination: The multiple timer model. Brain \& Cognition, 48, 117-132.

IVry, R. B., Spencer, R. M. C., ZelazniK, H. N., \& Diedrichsen, J. (2002). The cerebellum and event timing. In S. M. Highstein \& W. T. Thach, The cerebellum: Recent developments in cerebellar research (Annals of the New York Academy of Sciences, Vol. 978, pp. 302317). New York: New York Academy of Sciences.

Kelso, J. A. S. (1995). Dynamical patterns: The self-organization of brain and behavior. Cambridge, MA: MIT Press.

Kelso, J. A. S., Fink, P. W., Delaplain, C. L., \& Carson, R. G. (2001). Haptic information stabilizes and destabilizes coordination dynamics. Proceedings of the Royal Society of London: Series B, 268, 1207-1213.

Kelso, J. A. S., \& JEKA, J. J. (1992). Symmetry breaking dynamics of human multilimb coordination. Journal of Experimental Psychology: Human Perception \& Performance, 18, 645-668.

Kelso, J. A. S., Scholz, J. P., \& Schoner, G. (1986). Nonequilibrium phase transitions in coordinated biological motion: Critical fluctuations. Physics Letters, 118, 279-284.

KLAPP, S. T. (1981). Temporal compatibility in dual motor tasks II: Simultaneous articulation and hand movements. Memory \& Cognition, 9, 398-401.

Kunde, W., \& Weigelt, M. (2005). Goal congruency in bimanual object manipulation. Journal of Experimental Psychology: Human Perception \& Performance, 31, 145-156.

Mechsner, F. (2004a). Perceptual-cognitive control of bimanual coordination. International Journal of Sport \& Exercise Psychology, 2, 210-238.

Mechsner, F. (2004b). A psychological approach to human voluntary movements. Journal of Motor Behavior, 36, 355-370.

Mechsner, F., Kerzel, D., Knoblich, G., \& Prinz, W. (2001). Perceptual basis of bimanual coordination. Nature, 414, 69-73.

Monno, A., Temprado, J. J., Zanone, P. G., \& Laurent, M. (2002). The interplay of attention and bimanual coordination dynamics. Acta Psychologica, 110, 187-211.

Schmidt, R. C., Carello, C., \& Turvey, M. T. (1990). Phase transitions and critical fluctuations in the visual coordination of rhythmic movements between people. Journal of Experimental Psychology: Human Perception \& Performance, 16, 227-247.

Scholz, J. P., \& Kelso, J. A. S. (1990). Intentional switching between patterns of bimanual coordination depends on the intrinsic dynamics of the patterns. Journal of Motor Behavior, 22, 98-124.

Schoner, G., \& Kelso, J. A. S. (1988). Dynamic pattern generation in behavioral and neural systems. Science, 239, 1513-1520.

Semuen, A., \& Ivry, R. (2001). The coupled oscillator model of betweenhand coordination in alternate-hand tapping: A reappraisal. Journal of Experimental Psychology: Human Perception \& Performance, 27, 251-265.

Semjen, A., Summers, J. J., \& Cattaert, D. (1995). Hand coordination in bimanual circle drawing. Journal of Experimental Psychology: Human Perception \& Performance, 21, 1139-1157.

Spencer, R. M. C., Zelaznik, H. N., Diedrichsen, J., \& IVry, R. B. (2003). Disrupted timing of discontinuous movements by cerebellar lesions. Science, 300, 1437-1439.

Turvey, M. T. (1990). Coordination. American Psychologist, 45, 938-953.

Yamanishi, J., Kawato, M., \& SuzuKI, R. (1980). Two coupled oscillators as a model for the coordinated finger tapping by both hands. Biological Cybernetics, 37, 219-225. 


\section{NOTES}

1. We do not assume that this form of explicit timing is required for all rhythmic activities. For movements that do not entail salient points to define discrete cycles, temporal consistencies may be emergent, reflecting the operation of other control parameters (see Ivry et al., 2004).

2 . In pilot work with a different hand configuration, we observed that a number of subjects failed to spontaneously couple voice and hand movements. Because of this, we modified the instructions in the second half to encourage coupling.

3. We changed the movements because we wanted to ensure that phase transitions occurred on a significant percentage of the trials, and pilot testing indicated that phase transitions were more likely for abduction and adduction wrist movements than for flexion and extension movements.
4. The number of trials with at least three consecutive cycles with a relative phase $<135^{\circ}$ ( $M=6.88,4.7,7.5$ for the no-, one-, and twovocalization conditions, respectively) did not significantly differ across conditions $[F(2,22)=2.27, p=.13]$

5 . It is difficult to infer whether one event leads or follows another on the basis of observable events. For example, in synchronized tapping, the finger leads the metronome, although the phenomenal experience is that the events are coincident. Similarly, in our experiments we expect that the onset of vocalizations is coincident with some salient feature of the manual movements (e.g., wrist flexion), although the time at which such events are measurable may suggest a consistent phase lead of one response.

(Manuscript received October 5, 2004; revision accepted for publication September 18, 2005.) 